\title{
Conhecimento de acadêmicos de medicina sobre o câncer de colo de útero e conduta frente aos resultados da colpocitologia oncótica
}

\section{Knowledge of medical students about cervical cancer and management of oncotic colpocytology results}

Fernando Aquino de Sousa, Guilherme Martins Gomes Fontoura, Rafaela Cristina Araújo-Gomes, Paula Gabrielle Gomes Candido, Aloiso Sampaio Souza e Aldicleya Lima Luz.

\section{RESUMO:}

Introdução: A identificação de fatores de risco, diagnóstico e prevenção do câncer devem ser construídos e consolidados durante a formação médica. No entanto, os conteúdos de cancerologia ainda são limitados na maioria das escolas médicas, com estudantes considerando insatisfatória a atenção dada para a compreensão das doenças oncológicas na estrutura curricular. Objetivo: Avaliar e comparar o conhecimento e atitude dos acadêmicos de medicina acerca do câncer de colo de útero e da colpocitologia oncótica. Métodos: Os discentes foram separados em dois grupos: G1, com alunos do terceiro ano do curso, e G2, sexto ano. Aplicou-se questionário para avaliar e comparar o conhecimento dos grupos sobre os fatores de risco, sinais e sintomas do câncer cervical, atitudes diante do exame citopatológico e da percepção do ensino-aprendizagem de neoplasias cervicais na faculdade. Resultados: O G2 demonstrou compreenssão significativamente maior em relação aos sinais e sintomas ( $87,5 \%)$, afirmando que metrorragia e dor pélvica podem ser indícios da doença $(p=0,006)$, como também dispareunia e sangramentos durante 0 ato sexual $(91,1 \% ; p=0,043)$. Diante de um Papanicolau com diagnóstico de Lesão de Baixo Grau, 28,3\% do G1 solicitariam a repetição do exame em 6 meses, enquanto 53,7\% do $\mathrm{G} 2$ seguiriam essa conduta $(\mathrm{p}=0,012)$. A maioria dos estudantes $(45,3 \%)$ considera razoável a abordagem dos conteúdos sobre o câncer na faculdade e se sente parcialmente preparada $(49,2 \%)$ para atuar no controle da doença. Conclusão: Houve um ganho discreto nos conhecimentos do G2 em comparação ao G1, sinalizando que, apesar do maior contato e exposição aos conteúdos avaliados, esta progressão foi pouco representativa.

PALAVRAS-CHAVE: Neoplasias do Colo do Útero; Colo do útero; Aprendizagem Baseada em Problemas; Educação Médica.

\section{ABSTRACT:}

Introduction: The identification of risk factors, diagnosis and prevention of cancer must be constructed and consolidated during medical training. However, the content of cancerology is still limited in most medical schools, with students finding the attention given to understanding cancer diseases in the curriculum structure unsatisfactory. Objective: To evaluate and compare the knowledge and attitude of medical students about cervical cancer and oncotic colpocytology. Methods: The students were separated into two groups: G1, with students in the third year of the course, and G2, sixth year. A questionnaire was applied to assess and compare the knowledge of the groups on risk factors, signs and symptoms of cervical cancer, attitudes towards the cytopathological examination and the perception of teaching-learning of cervical neoplasms in college. Results: G2 demonstrated significantly greater understanding in relation to signs and symptoms (87.5\%), stating that metrorrhagia and pelvic pain may be indications of the disease $(p=0.006)$, as also dyspareunia and bleeding during sexual intercourse $(91.1 \% ; p=0.043)$. Faced with a Pap smear diagnosed with Low Grade Injury, $28.3 \%$ of $\mathrm{G} 1$ would request a repeat examination in 6 months, while $53.7 \%$ of $\mathrm{G} 2$ would follow this procedure $(p=0.012)$. Most students $(45.3 \%)$ consider the approach to cancer content in college to be reasonable and feel partially prepared $(49.2 \%)$ to act in controlling the disease. Conclusion: There was a slight gain in the knowledge of $\mathrm{G} 2$ compared to $\mathrm{G} 1$, signaling that, despite the greater contact and exposure to the contents evaluated, this progression was not representative.

KEYWORDS: Uterine Cervical Neoplasms; Cervix Uteri; Problem-Based Learning; Education, Medical. 


\section{INTRODUÇÃO}

O câncer cervical uterino (CCU) afeta mais de 570 mil mulheres no mundo, causando cerca de 311 mil mortes anuais, sendo $85 \%$ destas ocorridas em países pobres e em desenvolvimento ${ }^{1}$. Neste cenário, a doença ocupa, no Brasil, a terceira maior prevalência na população feminina, atingindo mais de 16 mil mulheres em 2018, com mortalidade superior a 5.700 casos naquele ano $^{2}$.

A infecção pelo papilomavírus humano (HPV) é o principal fator de risco e conditio sine qua non para 0 desenvolvimento do CCU invasivo ${ }^{3}$, e são conhecidos pelo menos 13 subtipos virais de alto risco carcinogênico ${ }^{4}$. Um estudo revelou que mais de $54 \%$ dos jovens brasileiros até 25 anos de idade estão infectados pelo HPV, e cepas de alto risco foram encontradas em até $38,4 \%$ dos indivíduos pesquisados 5 .

De curso clínico lento e assintomático em suas fases iniciais, o CCU é precedido por lesões intraepiteliais passíveis de detecção por exames ginecológicos, sendo a colpocitologia oncótica (também conhecida como exame citopatológico ou teste de Papanicolau) o método preconizado de rastreamento, recomendado para todas as mulheres entre 25 e 64 anos que tenham iniciado atividade sexual ${ }^{6}$.

A triagem e o diagnóstico precoce das lesões pré-malignas estão associados a um elevado potencial de cura e prevenção da doença invasiva, tendo valor imprescindível para as ações de prevenção primária e secundária, considerando que quase um terço das mulheres apresentam lesões cervicais induzidas pelo HPV já no primeiro ano de início da atividade sexual, e até $40 \%$ as apresentarão nos cinco anos subsequentes ${ }^{7}$.

O Papanicolau utiliza o esfregaço da cérvix uterino, e a citologia é caracterizada segundo os parâmetros do Sistema Bethesda, cujos resultados são classificados em negativo, atipias de células escamosas de significado indeterminado (ASC-US), atipias escamosas de significado indeterminado não excluindo lesão de alto grau (ASC-H), atipias de células glandulares (AGC), lesão intraepitelial de baixo grau (LSIL), lesão intraepitelial de alto grau (HSIL), adenocarcinoma in situ (AIC), e carcinoma invasor ${ }^{8}$.

Apesar de simples, eficaz e relativamente acessível, o Papanicolau tem sido alvo de controvérsias que envolvem desde erros na preparação dos esfregaços até a interpretação equivocada de seus resultados pelos profissionais de saúde, ocasionando dificuldades no diagnóstico e inadequado seguimento clínico de pacientes ${ }^{9}$. Visando à redução dessas falhas e garantia de melhorias no rastreamento, o Ministério da Saúde elaborou estratégias para as equipes de saúde responsáveis pelas ações de prevenção do CCU. Entre elas, o fortalecimento da formação e da qualificação profissional por meio de programas e metodologias de educação continuada ${ }^{10}$.

Os conhecimentos concernentes à epidemiologia, identificação de fatores de risco, diagnóstico e prevenção do câncer devem ser construídos e consolidados, indubitavelmente, durante a formação acadêmica, independentemente 
da especialidade a ser seguida ${ }^{11,12}$.

No entanto, os conteúdos de cancerologia ainda são limitados na maioria das escolas médicas, com uma importante parcela dos estudantes considerando insatisfatória a atenção dada para a compreensão das doenças oncológicas na estrutura curricular, o que, por sua vez, repercute na segurança autorreferida pelos discentes quanto às práticas de controle e enfrentamento das neoplasias, comparado a outras doenças comuns ${ }^{12,13}$.

Neste contexto, este estudo teve como objetivo avaliar e comparar o conhecimento de acadêmicos de medicina sobre o CCU e a conduta frente aos resultados do exame citopatológico.

\section{MÉTODO}

Estudo transversal e descritivo, realizado entre fevereiro e março de 2019, com os discentes do curso de medicina da Universidade Federal do Maranhão, campus Imperatriz. Foram convidados os acadêmicos do terceiro ano do curso, que compuseram o Grupo 1 (G1), e os acadêmicos do último ano (internato), que integraram o Grupo 2 (G2).

A estrutura curricular do curso baseia-se em metodologias ativas, com módulos temáticos interdisciplinares ao invés de disciplinas tradicionais. A escolha da amostra por acadêmicos do terceiro ano levou em consideração a conclusão do módulo de Proliferação Celular, que integra os conhecimentos sobre o cenário atual do câncer no Brasil e o estudo epidemiológico das principais neoplasias de interesse regional, enquanto a escolha pelos acadêmicos do último ano deu-se pelo maior tempo de observação e realização de diagnósticos e condutas sobre neoplasias cervicais durante 0 estágio ${ }^{14}$.

Os acadêmicos receberam um Termo de Consentimento Livre e Esclarecido (TCLE), conforme a Resolução $n^{0}$ 466/2012, do Conselho Nacional de Saúde, por meio do qual foi concedida permissão para a publicação dos resultados.

Utilizou-se um questionário com 30 questões objetivas, sendo cinco destinadas a caracterização sociodemográfica dos participantes, três direcionadas apenas as mulheres participantes em relação a suas práticas de prevenção, 17 questões específicas, elaboradas de acordo com as Diretrizes Brasileiras para Rastreamento do Câncer do Colo do Útero ${ }^{6}$, a fim de mensurar o conhecimento sobre o CCU e a conduta frente aos resultados, alterados ou não, do exame citopatológico, e cinco questões finais para avaliar a percepção dos discentes quanto ao processo de ensinoaprendizagem do câncer no âmbito acadêmico.

Os dados, organizados em planilhas, foram transportados para o programa Software Statistical Package for the Social Sciences - (SPSS), versão 20 e analisados por métodos de estatística descritiva. Como estratégia de comparação entre os G1 e G2 foi utilizado o teste do Qui-Quadrado, com intervalo de confiança de $95 \%$, com valor p significativo inferior a 0,05 .

O projeto de pesquisa foi submetido à Plataforma Brasil e aprovado pelo Comitê de Ética em Pesquisa da 
Universidade Federal do Maranhão sob parecer consubstanciado nº 3294117; CAAE: 08541018.8.0000.5087.

\section{RESULTADOS}

Participaram da pesquisa 126 acadêmicos, dos quais 46 (36,5\%) eram do G1, e 80 (63,5\%) eram do G2, ressaltando que o G1 possuí menos participantes devido compreender uma turma de medicina do $3^{\circ}$ ano, já o G2, foi composto por duas turmas, ambas do $6^{\circ}$ ano, estando no internato. Houve predomínio do sexo masculino (58,7\%), faixa etária entre 20 e 24 anos (56,3\%), e a maioria se autodeclarou parda (50,4\%) e solteira (84,8\%). Além disso, das 52 mulheres participantes do estudo, $76,9 \%$ já tinham sido submetidas ao exame citopatológico, onde $46,1 \%$ realizaram 0 último exame há menos de um ano e 82,7\% afirmaram terem sido orientadas sobre o "exame preventivo, HPV e CCU".

Os fatores de risco para o CCU e o HPV como agente intrínseco de sua patogênese foram bem identificados pelos acadêmicos, havendo concordância entre os dois grupos na maioria das respostas esperadas para cada item avaliado, exceto quanto à baixa escolaridade, em que as respostas divergiram significativamente $(p=0,012)$ entre $G 2$ e G1, conforme figura 1.

Figura 1: Avaliação do conhecimento sobre CCU.

Fatores de risco, sinais e sintomas

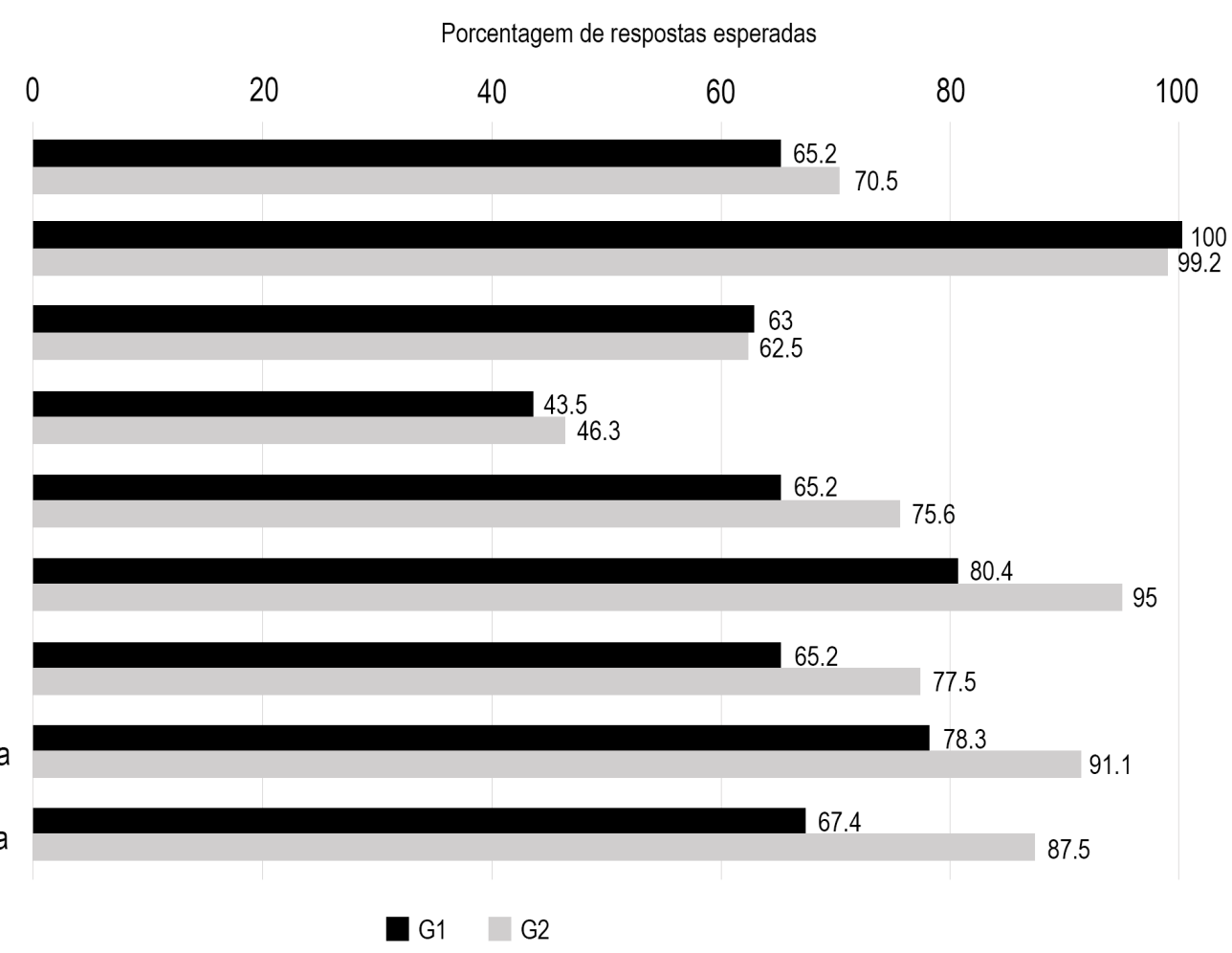

Legenda: Papilomavírus humano (HPV); Dispositivo intrauterino (DIU).

*Teste qui-quadrado $-p \leq 0,05$.

A análise dos principais sinais e sintomas, como sangramentos irregulares, dor e desconforto pélvico, revelou 
diferenças significativas entre os grupos, com incidência de acertos superior para o G2 (dispareunia/coitorragia - $p=0,043$; metrorragia/ dor pélvica $-p=0,006$ ). Manifestações incomuns, como febre e cefaleia, foram consideradas como anormais por $65,2 \%$ do G1 e $77,5 \%$ do G2 (Figura 1).

Em relação à colpocitologia oncótica, apenas $28,3 \%$ dos acadêmicos do G1 recomendariam a repetição do exame em 6 meses em caso de LSIL para mulheres de 25 anos ou mais, contra $53,7 \%$ do G2, sendo esta diferença estatisticamente significativa $(p=0,012)$. Em ambos os grupos, a colposcopia foi o método mais indicado como seguimento à investigação diagnóstica em todas as citologias cujos resultados fossem ASC-US, ASC-H, HSIL e AIC, observado na figura 2.

Figura 2: Conhecimento sobre colpocitologia oncótica.

Rastreamento e conduta frente ao exame citopatológico

Porcentagem de respostas esperadas

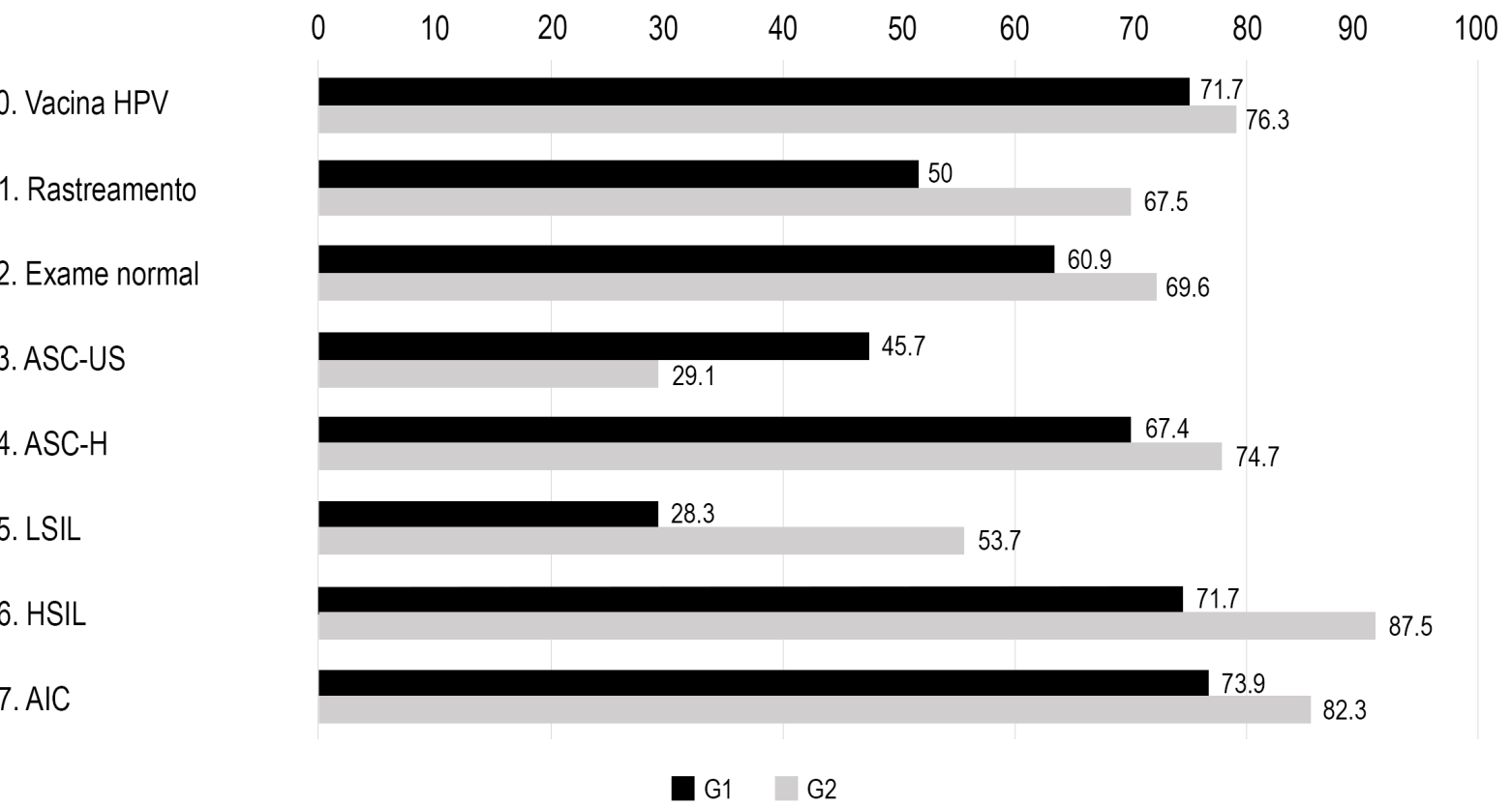

Legenda: Papilomavírus humano (HPV); Atipias de células escamosas de significado indeterminado (ASC-US); Atipias escamosas de significado indeterminado não excluindo lesão de alto grau (ASC-H); Lesão intraepitelial de baixo grau (LSIL); Lesão intraepitelial de alto grau (HSIL); Adenocarcinoma in situ (AIC).

*Teste qui-quadrado $-p \leq 0,05$.

A maior parte dos acadêmicos considera insuficiente (51,3\%/G2) ou razoável $(58,7 \% / G 1)$ o ensino e a abordagem do CCU ao longo da formação acadêmica, expressado pelo desejo prevalente entre os grupos (76,1\% de G1 e 70,5\% de G2) de que a grade curricular do curso ofertasse maior carga horária $(\mathrm{CH})$ aos módulos que compõem temas em oncologia. Além disso, 49,2\% dos participantes se sentem apenas parcialmente seguros em atuar na prevenção ao CCU. As demais informações sobre a percepção dos discentes sobre o ensino e inclusão dos temas em cancerologia estão contidas na tabela 1. 
Tabela 1: Percepção do ensino do CCU no Curso de Medicina da Universidade Federal do Maranhão, Campus Imperatriz/ MA.

\begin{tabular}{|c|c|c|c|}
\hline \multirow[b]{2}{*}{ Parâmetro } & \multicolumn{3}{|c|}{$\mathrm{n}(\%)$} \\
\hline & $\begin{array}{c}\mathrm{G1} \\
\mathrm{n}=46\end{array}$ & $\begin{array}{c}\mathrm{G} 2 \\
\mathrm{n}=80\end{array}$ & $\begin{array}{c}\text { Total } \\
n=126\end{array}$ \\
\hline \multicolumn{4}{|c|}{ Consideram o ensino/abordagem do $\mathrm{CCU}$} \\
\hline Suficiente & $4(8,7)$ & $9(11,5)$ & $13(10,3)$ \\
\hline Insuficiente & $15(32,6)$ & $41(51,3)$ & $56(44,4)$ \\
\hline Razoável & $27(58,7)$ & $30(37,2)$ & $57(45,3)$ \\
\hline \multicolumn{4}{|c|}{ Interesse pelo tema/cancerologia } \\
\hline Elevado & $5(10,9)$ & $11(13,7)$ & $16(12,7)$ \\
\hline Moderado & $28(60,9)$ & $42(52,6)$ & $70(55,5)$ \\
\hline Baixo & $13(28,3)$ & $27(33,7)$ & $40(31,8)$ \\
\hline \multicolumn{4}{|c|}{$\begin{array}{l}\text { Participação em atividades e/ou ligas de } \\
\text { cancerologia }\end{array}$} \\
\hline Sim & $26(56,5)$ & $60(75,6)$ & $86(68,2)^{*}$ \\
\hline Não & $20(43,5)$ & $20(24,4)$ & $40(31,8)$ \\
\hline \multicolumn{4}{|c|}{$\begin{array}{l}\text { Gostaria que o curso ofertasse maior } \mathrm{CH} \text { para } \\
\text { temas em cancerologia }\end{array}$} \\
\hline Sim & $35(76,1)$ & $57(70,5)$ & $92(73,0)$ \\
\hline Não & $10(21,7)$ & $16(20,5)$ & $26(20,6)$ \\
\hline Indiferente & $1(2,2)$ & $7(9,0)$ & $8(6,4)$ \\
\hline \multicolumn{4}{|c|}{ Segurança para atuar na prevenção do CCU } \\
\hline Sim & $10(21,7)$ & $42(52,6)$ & $52(41,3)$ \\
\hline Não & $4(8,7)$ & $8(10,3)$ & $12(9,5)$ \\
\hline Parcialmente & $32(69,6)$ & $30(37,2)$ & $62(49,2)$ \\
\hline
\end{tabular}

${ }^{*}$ Teste qui-quadrado $-p \leq 0,05$.

\section{DISCUSSÃO}

Identificar as bases fisiopatológicas, o curso clínico e as principais manifestações do CCU são imprescindíveis para a prática eficaz de promoção da saúde, prevenção e diagnóstico precoce desta e de outras neoplasias ${ }^{9}$. Apesar disso, a educação em oncologia e o ensino das neoplasias mais prevalentes ainda são deficitários em uma quantidade expressiva de escolas médicas, evidenciando uma abordagem fragmentada e pouco integrada entre a cancerologia e as demais áreas clínicas ${ }^{11,15}$. Nesse contexto, é legítimo avaliar o conhecimento acadêmico referente aos principais aspectos do câncer, de maneira a obter subsídios teóricos que sustentem a necessidade de melhorias estruturais dos currículos de graduação em medicina ${ }^{16,17}$.

Entre as mulheres que participaram deste estudo, a maioria informou ter sido orientada em consultas médicas anteriores sobre o exame preventivo e a vacina contra o HPV. Embora os dados do presente estudo representam o maior número de respostas, é importante notar que muitas mulheres não são rastreadas para o CCU ou imunizadas contra 
o HPV por não terem conhecimento da necessidade de realizar o exame ${ }^{18}$ ou da existência da vacina, atribuindo-se a isto a ausência de informações transmitidas por profissionais da saúde ${ }^{19}$. A "Carta aberta aos médicos", de coautoria da Federação Brasileira das Associações de Ginecologia e Obstetrícia (FEBRASGO), atenta para a seriedade com que os médicos, ginecologistas ou não, devem recomendar a vacinação contra o HPV, bem como oferecer, sem hesitação, informações contundentes a respeito da infecção pelo vírus e suas principais repercussões, como os tumores cervical, anal, vaginal, entre outros ${ }^{20}$.

O reconhecimento do HPV como agente causal e dos principais fatores de risco para o CCU foi semelhante entre os dois grupos. O G2, no entanto, obteve frequência de acertos significativamente maior que o G1 em relação à identificação dos sinais e sintomas mais comuns da doença, como a dispareunia, metrorragia e dor pélvica. Tal desempenho é similar ao do estudo de Silva et al., realizado com acadêmicos em uma faculdade de medicina em Goiânia $(G O)^{16}$, e superior ao de outras pesquisas, em que o índice médio de conhecimento entre estudantes de medicina sobre CCU foram expressamente menores ${ }^{21,22}$. Ademais, o estudo de Silva et al. ${ }^{16}$ demonstrou que essa diferença pode estar relacionada à melhora no desempenho dos acadêmicos ao longo do curso de medicina, tendo em seu estudo uma variação de acerto de 71,7\% (acadêmicos do primeiro semestre) até 83,2\% (acadêmicos do décimo segundo semestre), em relação aos conhecimentos acerca do HPV e CCU.

Quanto ao padrão alimentar e nutricional constituírem fator de risco para o desenvolvimento do câncer, pouco menos da metade de ambos os grupos do estudo julgou verdadeira esta correlação, sugerindo o predomínio de dúvidas quanto ao tema. A despeito da necessidade de pesquisas prospectivas para fundamentar, a nível fisiológico e molecular, a associação entre hábitos nutricionais e carcinogênese cervical, os achados de Kim et al. ${ }^{23}$ e Barchitta et al. ${ }^{24}$ apontam que a ingestão insuficiente de vitaminas antioxidantes presentes em frutas e vegetais está ligado a um maior risco de infecção por HPV de elevado risco oncogênico e progressão para o câncer.

A recomendação da vacina contra o HPV para crianças e adolescentes de ambos os gêneros foi indicada corretamente pela maioria dos participantes do estudo, não havendo divergências significativas na comparação entre os grupos. Estes resultados são consonantes às orientações do Ministério da Saúde ${ }^{25}$, e mostram-se superiores àqueles obtidos em outras pesquisas, em que cerca de $56 \%$ dos acadêmicos de medicina do último ano afirmaram que apenas as mulheres deveriam receber a vacina ${ }^{26}$. É oportuno salientar que $22,2 \%$ dos estudantes do presente estudo concordam que somente mulheres devam ser imunizadas, o que sinaliza atenção para maiores esforços em educação quanto ao tema, uma vez que os homens não vacinados possuem papel fundamental no ciclo de transmissão do HPV, enquanto infecção sexualmente transmissível.

A avaliação da atitude frente aos resultados da colpocitologia oncótica revelou diferenças significativas entre os grupos. Para apenas 28,3\% do G1, um Papanicolau com diagnóstico de LSIL deve ser repetido em 6 meses, enquanto $57,3 \%$ de $\mathrm{G} 2$ seguiriam esta mesma conduta $(p=0,012)$. Observou-se, adicionalmente, que os resultados do $\mathrm{G} 2$ 
demonstraram mais uniformidade em comparação aos do G1, embora, de todo modo, a maior parte de ambos os grupos tenha indicado adequadamente o seguimento nos casos de exames citopatológicos normais ou alterados, segundo as diretrizes estabelecidas pelo Instituto Nacional de Câncer José Alencar Gomes da Silva (INCA) ${ }^{6}$.

Compreender essas recomendações mostra-se indispensável, não apenas por refletir que o seguimento apropriado será dado às pacientes com alterações citopatológicas no colo do útero, mas também, por possibilitar uma difusão mais ampla do conhecimento a partir dos profissionais da saúde, por meio do maior acesso da população à informação. Gomes et al. constataram que 56,3\% das mulheres com câncer nunca se submeteram ao Papanicolau até o momento do diagnóstico, e apenas 12,6\% afirmou ter recebido orientações de médicos sobre a importância da prevenção, o que ilustra a dimensão da importância do dever médico em formar opinião e fomentar a saúde na atenção básica ${ }^{18}$.

Nesta pesquisa, enquanto uma fração significativa dos acadêmicos do G1 considera insuficientes os estudos sobre o câncer e a abordagem desse tema na faculdade, os do G2, por sua vez, acreditam que esses temas são retratados de forma razoável, o que sugere o provável incremento dos conteúdos relacionados a neoplasias ao longo dos períodos, sobretudo após o início do estágio curricular obrigatório (internato). A formação médica no Brasil, passou por mudanças consideráveis nas últimas duas décadas, com a instituição das Diretrizes Curriculares Nacionais do Curso de Graduação em Medicina ${ }^{27}$, que prevê a formação de um profissional generalista capaz de atuar na promoção de saúde, na prevenção e no diagnóstico das principais doenças humanas. Entretanto, o estudo de cancerologia não é suprido de maneira satisfatória na maioria das escolas médicas, criando lacunas na graduação, o que, invariavelmente, repercutem na qualidade do atendimento prestado à população, sendo esta dependente do preparo e capacitação desses futuros profissionais ${ }^{11-13,15}$. Por esta razão, as instituições de ensino superior do país devem contemplar, em seus currículos, 0 conhecimento integrado, contextualizado e voltado para a prevenção das doenças neoplásicas, tendo em vista o impacto do câncer na morbimortalidade da população brasileira e mundial ${ }^{1,2,4}$.

Esses achados são reforçados pelo fato de a maioria dos discentes de G1 acreditarem que, ao sair da faculdade, estarão pouco ou parcialmente preparados para atuarem nas ações de prevenção e controle do câncer, em contraposição aos alunos dos semestres finais (G2), que afirmaram sentir-se mais seguros e preparados. Contudo, mesmo entre os acadêmicos do G2, uma quantia considerável ainda julga estar pouco ou moderadamente preparada, indo ao encontro de resultados semelhantes em outros estudos que investigaram os sentimentos vividos por alunos concernentes à prática clínica ${ }^{28,29}$.

\section{CONSIDERAÇÕES FINAIS}

A maioria das variáveis avaliadas apresentaram percentual de respostas esperadas maior que $50 \%$ em ambos 
os grupos, o que indica bom conhecimento no geral. Contudo, esses conhecimentos devem ser aprimorados, visto que, quando formados, os participantes de ambos os grupos prestarão assistência dentro dessa temática, e necessitarão estar preparados para esse atendimento.

Na comparação do conhecimento entre os grupos, verificou-se que o G2 apresentou melhor aproveitamento, estatisticamente significativo, para 3 variáveis, e de modo geral representado nas médias de 14 das 17 variáveis analisadas. O que pode ser justificado devido a uma maior exposição desses participantes sobre os conteúdos avaliados

dentro da prática clínica dos estágios e do próprio internato. É necessário aprimorar o ensino do CCU na graduação médica de modo a formar profissionais seguros e capazes de compreender a integralidade dessa doença neoplásica no contexto da atenção primária à saúde.

\section{REFERÊNCIAS}

1. Bray F, Ferlay J, Soerjomataram I, Siegel RL, Torre LA, Jemal A. Global cancer statistics 2018: GLOBOCAN estimates of incidence and mortality worldwide for 36 cancers in 185 countries. CA Cancer J Clin. 2018;68(6):394-424.

2. Instituto Nacional de Câncer José Alencar Gomes da Silva (INCA) [Internet]. Estimativa 2018: Incidência de câncer no Brasil. Rio de Janeiro: INCA; 2018. 130 p. Disponível em: https://www.inca.gov.br/sites/ufu.sti.inca.local/files// media/document//estimativa-incidencia-de-cancer-no-brasil-2018.pdf. Acesso em: 09 de nov de 2020

3. Barukčić I. Human Papillomavirus — The Cause of Human Cervical Cancer. J Biosci Med. 2018;06(04):106-25.

4. De Martel C, Plummer M, Vignat J, Franceschi S. Worldwide burden of cancer attributable to HPV by site, country and HPV type. Int J Cancer. 2017;141(4):664-70.

5. Estudo Epidemiológico sobre a Prevalência Nacional de Infecção pelo HPV (POP-Brasil): Resultados preliminares [Internet]. Associação Hospitalar Moinhos de Vento. Porto Alegre, 2017. Disponível em: www.iepmoinhos.com.br/ pesquisa/downloads/LIVRO-POP_Brasil_-_Resultados_Preliminares.pdf. Acesso em 20 nov de 2020

6. Instituto Nacional de Câncer José de Alencar Gomes da Silva [Internet]. Divisão de Detecção Precoce e Apoio à Organização de Rede. Diretrizes Brasileiras para o Rastreamento do Câncer do Colo do Útero. INCA; 2016. 118 p. Disponível em: https://www.inca.gov.br/sites/ufu.sti.inca.local/files//media/document/diretrizesparaorasdiretrizesparaorastreamentodoc_2016_corrigido.pdf. Acesso em 04 de dez de 2019

7. Monteiro DLM, Trajano AJB, Silva KS, Russomano FB. Incidence of cervical intraepithelial lesions in a population of adolescents treated in public health services in Rio de Janeiro, Brazil. Cad Saude Publica. 2009;25(5):1113-22.

8. Solomon D, Davey D, Kurman R, Moriarty A, O'Connor D, Prey M, et al. The 2001 Bethesda System: Terminology for reporting results of cervical cytology. J Am Med Assoc. 2002;287(16):2114-9. 
9. Amaral AF, Araújo ES, Magalhães JC, Silveira ÉA, Tavares SBN, Amaral RG. Impacto da capacitação dos profissionais de saúde sobre o rastreamento do câncer do colo do útero em unidades básicas de saúde. Rev Bras Ginecol e Obs. 2014;36(4):182-7.

10. Instituto Nacional de Câncer José de Alencar Gomes da Silva [Internet]. Divisão de Apoio à Rede de Atenção Oncológica. Plano de Ação para Redução da Incidência e Mortalidade por Câncer do Colo do Útero. INCA; 2010. 42 p. Disponível em: https://www.inca.gov.br/sites/ufu.sti.inca.local/files//media/document/plano-de-acao-para-reducao-da-incidencia-e-mortalidade-por-cancer-do-colo-do-utero-2010.pdf. Acesso em 13 de dez de 2019.

11. Silvestrini AA, Scherrer LR, Moreira WB. O ensino de oncologia na graduação: panorama brasileiro. Revista Brasileira de Oncologia Clínica. 2012;8:125-9.

12. Neeley BC, Golden DW, Brower J V., Braunstein SE, Hirsch AE, Mattes MD. Student Perspectives on Oncology Curricula at United States Medical Schools. J Cancer Educ. 2019;34(1):56-8.

13. Ferreira DAV., Da Silva AP, Da Silva KRX. Ensino de oncologia na graduação médica e autorregulação da aprendizagem. Rev Hosp Univ Pedro Ernesto. 2015;14(0):50-8.

14. Projeto pedagógico do curso de medicina de Imperatriz-MA [Internet]. Universidade Federal do Maranhão. 2017. 248 p. Disponível em: https://sigaa.ufma.br/sigaa/public/curso/documentos_curso.jsf?lc=pt_BR\&id=12125248. Acesso em 20 de fev de 2019.

15. Amgad M, Shash E, Gaafar R. Cancer education for medical students in developing countries: Where do we stand and how to improve? Crit Rev Oncol Hematol. 2012;84(1):122-9.

16. Silva SL da, Vargas AL, Almeida RJ, Saddi VA, Cordeiro JABL, Silva AMTC. Conhecimento dos acadêmicos de medicina acerca do HPV e do câncer de colo uterino. Saúde (Santa Maria). 2017;43(2):125.

17. Rodriguez-Feria P, Hernández-Florez LJ, Rodriguez-Feria D. Knowledge, attitudes and practices of prevention for cervical cancer and breast cancer among medical students. Rev Salud Pública. 2016;18(3):354.

18. Gomes CHR, Silva JA Da, Ribeiro JA, Penna RMM. Câncer Cervicouterino: Correlação entre diagnóstico e realização prévia de exame preventivo em serviço de referência no norte de Minas Gerais. Rev Bras Cancerol. 2012;58(1):41-5.

19. Pereira RGV, Machado JLM, Machado VM, Mutran TJ, Santos LS dos, Oliveira E, et al. A influência do conhecimento na atitude frente à vacina contra o Papilomavírus Humano: ensaio clínico randomizado. ABCS Heal Sci. 2016;41(2):78-83.

20. Federação Brasileira das Associações de Ginecologia e Obstetrícia [Internet]. Carta aberta aos médicos: Vacina HPV. São Paulo: FEBRASGO, set. 2015. Disponível em: https://sbim.org.br/images/files/carta_medicos_ hpv_10092015.pdf. Acesso em 23 de jun de 2020. 
21. Adejuyigbe FF, Balogun BR, Sekoni AO, Adegbola AA. Cervical cancer and human papilloma virus knowledge and acceptance of vaccination among medical students in Southwest Nigeria. Afr J Reprod Health. 2013;19(1):140-8.

22. Akpo D, Deji P, Idiake V, Otohinoyi D, Medavarapu S. Cervical cancer: knowledge, screening practices and vaccines among female medical students in the Commonwealth of Dominica. Arch Med. 2016;8(5):1-5.

23. Kim J, Kim MK, Lee JK, Kim JH, Son SK, Song ES, et al. Intakes of vitamin A, C, and E, and -carotene are associated with risk of cervical cancer: A case-control study in Korea. Nutr Cancer. 2010;62(2):181-9.

24. Barchitta M, Maugeri A, Quattrocchi A, Agrifoglio O, Scalisi A, Agodi A. The association of dietary patterns with high-risk human papillomavirus infection and cervical cancer: A cross-sectional study in Italy. Nutrients. 2018;10(4).

25. Informe técnico da ampliação da oferta das vacinas papilomavírus humano 6, 11, 16 e 18 (recombinante) vacina HPV quadrivalente e meningocócica C (conjugada) [Internet]. Brasil. Ministério da Saúde. Secr Vigilância em Saúde. Dep Vigilância das Doenças Transm. Coord Programa Nac Imunizações. 2018. 39 p. Disponível em: http://portalarquivos2.saude.gov.br/images/pdf/2018/marco/14/Informe-T--cnico-HPV-MENINGITE.pdf. Acesso em 20 de jun de 2020

26. Monteiro DLM, Brollo LCS, Souza TP, Santos JRP, Santos GR, Correa T, et al. Knowledge on the HPV vaccine among university students. Rev Inst Med Trop Sao Paulo. 2018;60(July):1-8.

27. Diretrizes Curriculares Nacionais do Curso de Graduação em Medicina e dá outras providências [Internet]. Brasil. Ministério da Educação. Conselho Nacional de Educação. Resolução № 3, de 20 de junho de 2014. 2014. 14 p. Disponível em: http://portal.mec.gov.br/index.php?option=com_docman\&view=download\&alias=15874-rces003-14\&category_slug=junho-2014-pdf\&ltemid=30192. Acesso em 20 de fev de 2019.

28. Kaluf IO, Gurgel S, Sousa O, Luz S, Cesario RR. Sentimentos do estudante de medicina quando em contato com a Prática. Rev Bras Educ Med. 2019;43(1):13-22.

29. Freitas LS, Ribeiro MF, Barata JLM. O desenvolvimento de competências na formação médica: os desafios de se conciliar as Diretrizes Curriculares Nacionais num cenário educacional em transformação. Rev Médica Minas Gerais. 2018;28:1-8. 